\title{
Medial Patellofemoral Ligament Reconstruction and Nonanatomic Stabilization Techniques in Skeletally Immature Patients
}

\author{
Carola Pilone ${ }^{1}$ Davide Edoardo Bonasia ${ }^{1}$ Federica Rosso ${ }^{1}$ Umberto Cottino ${ }^{1}$ Claudio Mazzola ${ }^{2}$ \\ Davide Blonna ${ }^{1}$ Roberto Rossi ${ }^{1}$ and Sigascot Knee Committee \\ 1 Department of Orthopaedics and Traumatology, AO Ordine \\ Mauriziano Hospital, University of Torino, Torino, Italy \\ 2 SC Ortopedia Delle Articolazioni, Ospedali Galliera Genova, Genoa, Italy

\begin{abstract}
Address for correspondence Davide Edoardo Bonasia, MD, Department of Orthopaedics and Traumatology, AO Ordine
\end{abstract} \\ Mauriziano Hospital, University of Torino, Via Lamarmora 26, \\ 10128 Torino, Italy (e-mail: davidebonasia@virgilio.it).
}

Joints 2019;7:98-106.

\begin{abstract}
Keywords

- medial patellofemoral ligament

- children

- adolescent

- MPFL

- patellar instability

Patellar instability is a common cause of knee disability in children and adolescent, with a high recurrence rate. When conservative treatment fails, surgical options should be considered. The femoral insertion of the medial patellofemoral ligament (MPFL) is in close proximity to the distal femoral growth plate and precautions should be taken to avoid injuries to the physis. Anatomical features of the MPFL complex, with focus on the relationship between femoral MPFL attachment and femoral physis, are discussed together with surgical tips to avoid injuries to the growth plates. The aim of this article is to review the recent literature regarding MPFL reconstruction and other stabilization techniques for patellofemoral instability in skeletally immature patients, focusing on the different surgical options available. These can be classified as anatomical versus nonanatomical, proximal versus distal realignments, or based on the graft used: free graft and pedicled graft (quadriceps, patellar tendon, hamstring, and adductor magnus).
\end{abstract}

\section{Introduction}

Patellar instability is a common cause of knee disability in children and adolescent. ${ }^{1}$ After the first episode of dislocation, approximately 7 to $71 \%$ of patients experience symptoms and recurrent instability. ${ }^{2}$

Weeks et al proposed a classification for patellofemoral instability in children and adolescents, including four types: posttraumatic, syndromic, obligatory, and fixed. ${ }^{3}$

The most common type is traumatic dislocation, subdivided into first time and recurrent. In obligatory dislocations, patients dislocate at every knee flexion with self-reduction in extension and this type is typically associated with a shortened extensor mechanism and tight lateral retinaculum. In fixed lateral dislocation, the patella is irreducible at any degree of knee flexion, and is often associated with other limb deficiency. Syndromic dislocations have a genetic predis-

received

January 12, 2018 accepted after revision

October 15, 2019

published online

December 13, 2019 position to instability due to connective disorders (i.e., Marfan syndrome, Ehlers-Danlos syndrome, Down syndrome, Nailpatella syndrome, and skeletal dysplasia). ${ }^{3}$

Syndromic, obligatory, and fixed lateral dislocations show anatomic variants of lower limb (such as patella alta, trochlear dysplasia, hyperlaxity, increased $Q$ angle, and torsional deformities). For these patients, the treatment includes medial patellofemoral ligament (MPFL) reconstruction associated with the correction of the underlying pathology: distal realignment (for increased $Q$ angle or TT-TG distance), lateral release, quadriceps lengthening, or osteotomies (derotational or varus). ${ }^{3,4}$

After a first lateral patellar dislocation, MPFL injuries occur in more than $90 \%$ of patients. ${ }^{5}$ The MPFL is the main static stabilizer of the patella and MPFL reconstruction is the most common surgical treatment for patellar instability in adult and pediatric patients. However, the femoral insertion

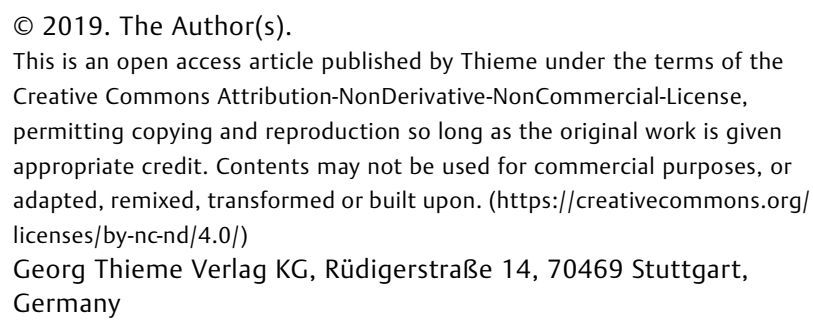


of the MPFL is in close proximity to the distal femoral growth plate and precautions should be taken to avoid injuries to the physis.

The aim of this article is to review the recent literature regarding MPFL reconstruction in skeletally immature patients, focusing on the surgical techniques available.

\section{Anatomy}

The MPFL represents the major static stabilizer of patellofemoral joint and at 0 to 30 degrees of knee flexion is the only restraint against patellar lateralization. ${ }^{5}$ With increased knee flexion, the patella is engaged in the trochlea and bone morphology becomes the main stabilizer. ${ }^{5}$

The MPFL origins close to the medial femoral epicondyle and inserts between the proximal and mid-third of the medial border of the patella. According to the three-layer classification of the medial compartment described by Warren and Marshall, the MPFL is located in the second layer. ${ }^{6}$ The femoral origin is distal to the adductor tubercle and posterosuperior to the medial epicondyle. ${ }^{7}$ LaPrade et al, in an anatomical study, identified the MPFL femoral insertion $10.6 \mathrm{~mm}$ proximal and $8.8 \mathrm{~mm}$ posterior to the medial epicondyle and $10 \mathrm{~mm}$ anterior and $3.8 \mathrm{~mm}$ distal to the adductor tubercle. ${ }^{8}$

Schottle et al $^{9}$ described a radiographic method to identify the landmark for MPFL femoral insertion on a true lateral X-ray view of the knee, also known as the "Schottle point." This point is located $1 \mathrm{~mm}$ anterior to the posterior femoral cortex, $2.5 \mathrm{~mm}$ distal to the origin of medial condyle, and proximal to the most posterior point of Blumensaat's line. ${ }^{9}$

In the pediatric population, the MPFL origin is in close proximity to the distal femoral physis and many papers studied the relationship between the two structures.

Nelitz et al $^{10}$ analyzed 27 anteroposterior (AP) and true lateral radiographs of skeletally immature patients with patellar instability. The authors found that the projected median origin of the MPFL as measured on a lateral radiograph was located $3.2 \mathrm{~mm}(1.2-5.8 \mathrm{~mm})$ proximal to the physis. The median distance between the most medial part of the physis and the physeal line on the AP radiograph was $9.9 \mathrm{~mm}$ (4.1-12.0 mm). Subtracting the two measured values, the median origin of the MPFL as seen on the AP view was $6.4 \mathrm{~mm}(2.9-8.5 \mathrm{~mm})$ distal to the femoral physis. The authors also suggested that determining the MPFL origin only with Schottle method can cause physeal injuries. Shea et $\mathrm{al}^{11}$ reported, in an indirect radiographic study using a lateral X-ray, that the insertion of the MPFL was 2 to $5 \mathrm{~mm}$ proximal to the distal femoral physis.

Farrow et al ${ }^{12}$ confirmed Nelitz et al's ${ }^{10}$ results in a study based on 16 skeletally immature cadaveric specimens. In all specimens MPFL femoral origin was distal to the medial border of the femoral physis. The medial aspect of the physis was concave posteriorly and convex anteriorly and a MPFL insertion was distal to the concave part and proximal to the convex one. The authors have also investigated the relationship between physis and femoral tunnel for MPFL anatomic reconstruction. They suggested angling the tunnel distal and anterior to decrease the risk of distal growth plate damage. ${ }^{12}$
A recent study by Nguyen et $\mathrm{al}^{13}$ analyzed the safe angle for drilling the distal femoral epiphysis in MPFL reconstruction. The authors studied 23 three-dimensional images of cadaveric specimens simulating drill paths for the placement of a $6-\mathrm{mm}$ interference screw. The safest tunnel was $20 \mathrm{~mm}$ long with an angulation of 15 to 20 degrees both distally and anteriorly. Changes of angulation in sagittal and coronal plane of 5 degrees in each direction decreased the percentage of safe tunnels. ${ }^{13}$

Therefore, as the femoral origin of MPFL seems to be located distally to distal femoral physis, a safe drill path for reconstruction has to be angulated at 15 to 20 degrees distally and anteriorly. Determining the insertion point only using lateral radiographs is not safe and also an AP view is recommended.

\section{Conservative Treatment}

The first episode of patellar dislocation can be treated conservatively. After patellar relocation, the knee is immobilized in extension with a brace for 3 weeks to allow for MPFL and other medial structures healing. The reason for the high reparative potential of the MPFL with conservative treatment is that, in adolescents, ligaments mostly tear off their insertion, whereas in adults the tear is generally midsubstance. ${ }^{1}$ Indications for surgery at first dislocation include large bony avulsions or loose chondral fragments amenable to repair.

Patellar insertion is the most common zone of MPFL injury in children. In 43 magnetic resonance imaging scans, Kepler et $\mathrm{al}^{14}$ found that MPFL was injured at patellar insertion in $61 \%$ of patients, at femoral insertion in $20 \%$, at both patellar and femoral insertion in $12 \%$, and at multiple locations in $15 \%$.

However, conservative treatment is associated with a $69 \%$ recurrence rate. ${ }^{15}$ In a systematic review regarding surgical versus conservative treatment of acute dislocations, Nwachukwu et al ${ }^{16}$ noted that conservatively managed knees had a $31 \%$ recurrence rate, compared with $22 \%$ in the surgical group (mostly acute repair). Also, better clinical outcomes were found in the surgical group.

\section{Patellofemoral Stabilization Techniques}

Surgical treatment is indicated in case of associated osteochondral injuries or failure of conservative treatment.

All techniques available have in common the goal of preserving the distal femoral physis. These can be classified as anatomical versus nonanatomical, proximal versus distal realignments, or based on the graft used. In the present paper, the different surgical techniques have been divided according to the graft choice in: free graft and pedicled graft (quadriceps, patellar tendon, hamstring, and adductor magnus).

\section{Free Graft Techniques}

Free graft techniques are generally anatomic and performed with a free soft tissue graft. Autologous gracilis tendon (or semitendinosus tendon) are the most commonly used 
autografts. Alternatively, soft tissue allografts can be used. ${ }^{17}$ Generally, the graft has two patellar fixation points and one femoral fixation point. The fixation technique varies among the different studies.

\section{Schottle's Technique}

A free gracilis autograft is harvested through a longitudinal or oblique anteromedial incision centered on the pes anserinus. A2- to 3-cm skin incision is performed starting from the superomedial margin of the patella to the patellar midpoint. The bony insertion area of the MPFL is prepared and a bony trough of $2 \mathrm{~cm}$ is created with a rongeur. Two 4.2- $\mathrm{mm}$ suture anchors with no. 2 braided sutures are placed on the medial aspect of the patella. The second and the third layer of the medial patellofemoral complex are separated down to the femoral insertion. A 2-cm longitudinal skin incision is made from the medial epicondyle to the adductor tubercle. An eyelet guidewire is placed slightly posterior to the midpoint between the medial epicondyle and the adductor tubercle. In the pediatric population, the guidewire should be inserted with anterior and distal angulation, as previously described. Guidewire placement is checked with fluoroscopy, on a perfect lateral view. The femoral attachment should be located slightly anterior to the line drawn on the posterior femoral cortex and between two lines (perpendicular to the posterior cortex line) drawn on the proximal origin of the medial condyle and the most posterior point of the Blumensaat's line. The guidewire is overdrilled with a cannulated drill tip $0.5 \mathrm{~mm}$ larger than the size of the two free ends of the graft. Attention should be paid not to violate the lateral wall of the medial femoral condyle in the intercondylar notch. A shuttle suture is placed in the femoral tunnel with the eyelet guidewire and retrieved from the lateral aspect of the knee. The midpoint of the graft is then fixed in the patellar trough with the sutures of the suture anchors. The free ends of the graft are then retrieved from the femoral incision, between layers 2 and 3 . The length of the free ends required to fill almost all the femoral tunnel is measured, and the graft trimmed accordingly. The free ends are then armed together with a no. 2 absorbable suture and the graft is pulled into the femoral socket with the previously placed shuttle suture. Fixation is achieved with a bioabsorbable interference screw at 30 degrees of knee flexion. Full range of motion is checked together with patellar tracking. Overtightening should be avoided ( $\mathbf{F i g . 1 A}){ }^{9}$

\section{Nelitz's Technique}

In Nelitz's technique, ${ }^{18}$ a $4-\mathrm{mm}$ drill is used to create two converging tunnels in the patella (without injuring the cortical bone bridge), to obtain a V-shaped tunnel at the superomedial half of the patella. Sufficient distance between tunnels of approximately $1 \mathrm{~cm}$ is necessary to avoid fractures. A free gracilis graft can then be inserted into the tunnel forming a loop through the patella. The Schottlel's technique ${ }^{9}$ is used to determine the femoral attachment of the MPFL on the lateral view. However, the authors highlight that the lateral view alone can be misleading for the determination of the relation between the distal femoral physis and the MPFL.
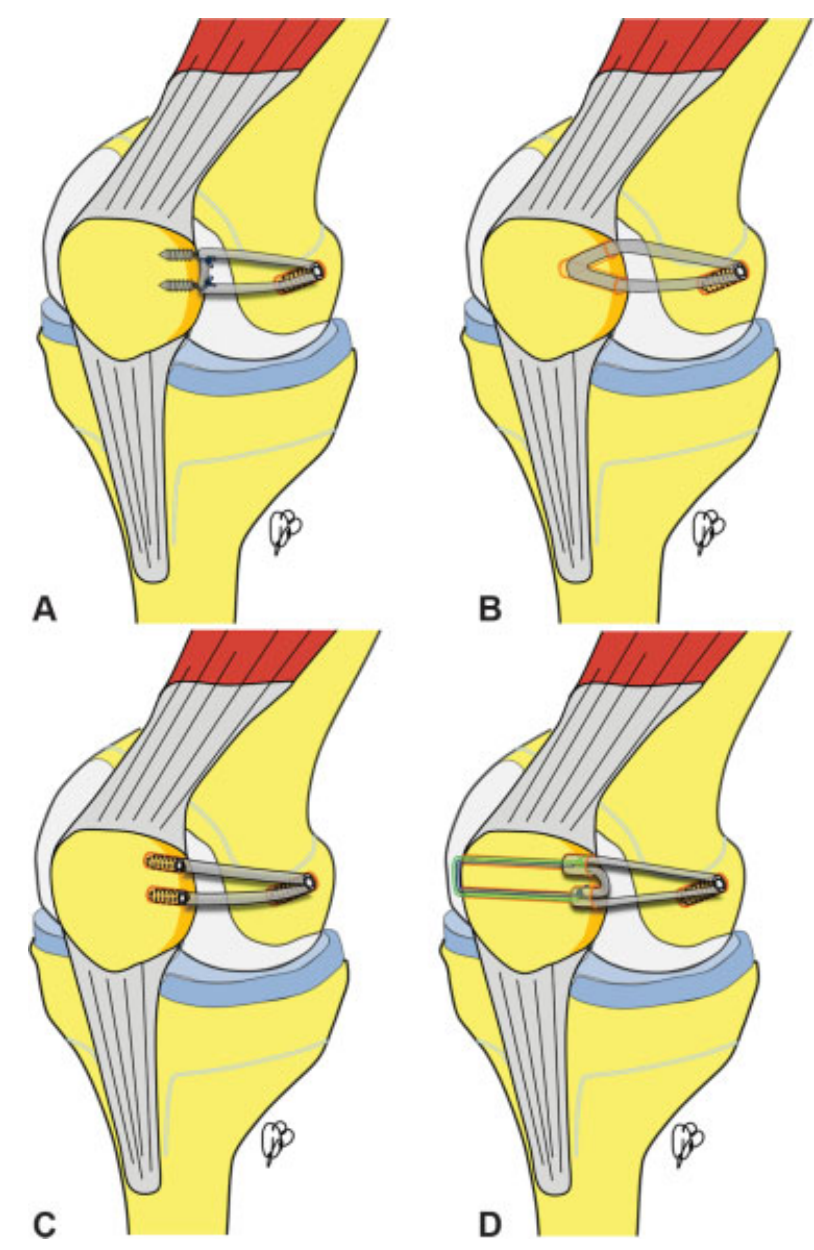

Fig. 1 Free graft techniques: (A) Schottle et al's technique; (B) Nelitz's technique; (C) Ladenhauf's technique; and (D) Shafizadeh's technique.

The authors underlined that the cross-reference onto an AP view shows that the same point that is projected on or proximal to the physis on the lateral view is actually distal to the physis on the AP view. When the intraoperatively measured distance is $<5 \mathrm{~mm}$, the guide pin has to be directed more distally, to preserve the physis. The graft is then secured within the medial condyle tunnel using a bioresorbable interference screw with the knee flexed at 30 degrees (-Fig. 1B). ${ }^{18}$

\section{Ladenhauf's Technique}

In Ladenhauf et al's technique, ${ }^{19}$ a free gracilis or semitendinosus autograft is harvested. The femoral insertion point is identified as previously described. A $15-$ to $20-\mathrm{mm}$ long, 6-mm diameter femoral tunnel is drilled and the previously harvested double-bundled autograft is secured with a tenodesis screw. The two free ends are passed subcutaneously beneath the fascia but superficial to the joint capsule. Two 15-mm long (4.5-mm diameter) socket tunnels are drilled in the patella. The appropriate length of the free ends is determined and the graft trimmed accordingly. With the patella in the center of the trochlea and the knee at 30 degrees of flexion, the free ends of the graft are fixed with tenodesis screws in the patellar sockets ( - Fig. 1C). ${ }^{19}$ 


\section{Shafizadeh's Technique}

Shafizadeh and Balke ${ }^{20}$ described an implant-free patellar fixation technique for MPFL reconstruction, with a free gracilis tendon. After exposing the medial aspect of the patella and creating a small groove, a $2.4-\mathrm{mm}$ eyelet wire is drilled through the patella from medial to lateral. With a cannulated $4.5-\mathrm{mm}$ drill, a tunnel with a depth of $1 \mathrm{~cm}$ is drilled over the wire. A looped no. 2 nonabsorbable, braided suture is inserted through the eyelet of the wire in a loop configuration. The wire is then pierced through the skin. A second similar drill hole is made more distally, and a second suture is passed with the same technique. The length of the bone bridge between both holes should be around $15 \mathrm{~mm}$. Next, one suture is passed through the loop of the other suture, and pulled medially. At this point, one U-shaped suture should run through both drill holes over the lateral bone bridge. Two opposed high strength sutures (the loop of one suture and both free ends of the other suture) are passed through the loop of the shuttle suture, and pulled around the lateral bone bridge medially through the drill holes. The graft is passed through both loops. By pulling on the free ends of both sutures, the graft is pulled press-fit into the $1-\mathrm{cm}$ bone sockets. Fixation of the graft is achieved by tying the free ends of the sutures over the graft at the proximal and distal bone tunnels. Femoral tunnel creation and fixation are as described by Schottle et al ( - Fig. 1D). ${ }^{9}$

\section{Sadigursky's Technique}

Sadigursky et $\mathrm{al}^{21}$ described a technique very similar to Ladenhauf's, ${ }^{19}$ but the free ends of the graft are fixed to the patella with two suture anchors (instead of two interference screw in the patellar sockets) ( - Fig. 2A). ${ }^{21}$

\section{Authors' Preferred Technique}

The authors' favorite technique is a variation of Schottle's ${ }^{9}$ and Shafizadeh's ${ }^{20}$ techniques. A free gracilis graft is harvested. The medial aspect of the patella is prepared as described by Schottle et al, then two $2.5-\mathrm{mm}$ eyelet guidewires are inserted in the patella from medial to lateral, making sure to have around $1 \mathrm{~cm}$ of bone bridge on the lateral patellar aspect. No tunnels or half sockets are drilled in the patella. A looped no. 5 nonresorbable suture is passed through the $2.5-\mathrm{mm}$ tunnels. The graft is then fixed to the patella with the no. 5 suture. The femoral incision is then performed, as described by Schottle et al..$^{9}$ A $20-\mathrm{mm}$ long femoral tunnel (same diameter of the two free ends of the graft) is drilled in the Schottle's point, ${ }^{9}$ making sure with image intensifier to preserve the growth plates. Both lateral and AP views are used. Femoral fixation is achieved with an interference screw. When the isometric point is too close to the growth plate or in younger patients, a suture anchor is used for fixation (-Fig. 2B).

\section{Kumahashi's Technique}

Kumahashi et $\mathrm{al}^{22}$ described the "sandwich" technique for MPFL reconstruction. One end of a free semitendinosus graft is fixed to the anatomical insertion on the patella in a bone tunnel with an interference screw. The other end of the graft
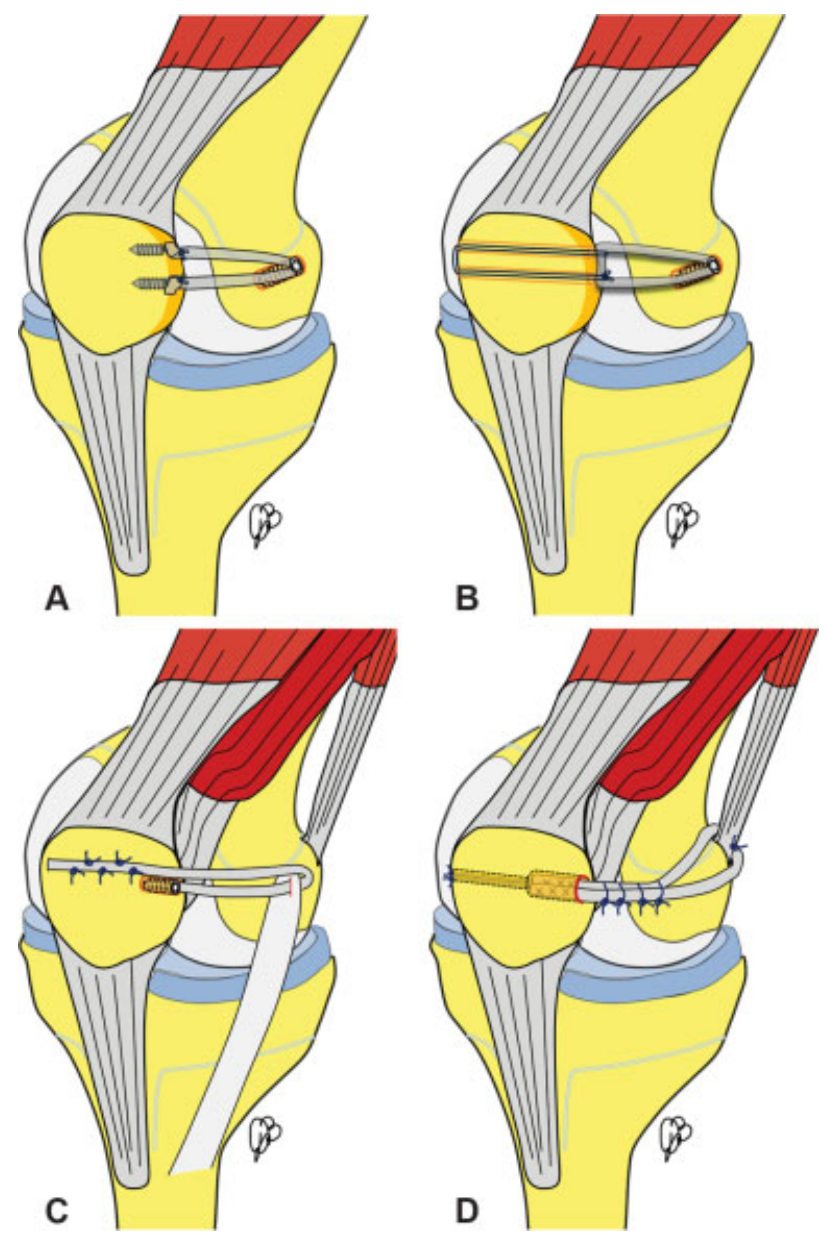

Fig. 2 Free graft techniques: (A) Sadigursky's technique; (B) Authors' preferred technique; (C) Kumahashi's technique; and (D) Gomes' technique.

is then passed between layers 2 and 3, looped around medial collateral ligament (MCL) as a pulley, and then sutured on the anterior aspect of the patella ( $\mathbf{F i g . ~ 2 C )}{ }^{22}$

\section{Nonanatomic Free Graft Techniques}

\section{Adductor Sling}

Yercan et $\mathrm{al}^{23}$ described a technique with a free semitendinosus tendon for MPFL reconstruction. Two converging patellar tunnel are created. The first patellar tunnel is created with $4.5-\mathrm{mm}$ drill at the mid aspect of the medial patella with an AP and proximal-distal direction. The second tunnel is created with $3.2-\mathrm{mm}$ drill and the entry point is localized at the center of the patella. The semitendinosus is passed into the tunnel, and then between layers 2 and 3 of the medial retinaculum. Tenodesis to the adductor tendon is performed for femoral fixation. In younger children, the authors suggested the creation of a pulley from the fascia on the anterior patellar surface, instead of drilling patellar tunnels. ${ }^{23}$

In Gomes's technique ${ }^{24}$ (-Fig. 2D), a $10-\mathrm{mm}$ incision is made in the posteromedial aspect of the knee over the hamstring. The semitendinosus tendon is identified, pulled out, and examined. If strong enough, it is split, and one-half is harvested. Otherwise the whole tendon is harvested. The 
patella is transfixed in the middle line, from lateral to medial, with a $2.7-\mathrm{mm}$ drill inserted from the anteromedial arthroscopic portal. On the medial side, the diameter of the drilled tunnel is enlarged to $3.2 \mathrm{~mm}$ in the first $10 \mathrm{~mm}$. An osteoperiosteal tunnel is drilled under the adductor magnus at its distal femoral insertion. One graft end is passed through this tunnel and folded over itself. The arming sutures at the ends of the graft are passed through the patellar tunnel, exiting from the lateral side of the patella. With the knee flexed at 60 degrees, the arming sutures are fixed to the lateral retinaculum. ${ }^{24}$

A relatively similar technique was described by Lind et al. ${ }^{25} \mathrm{~A}$ free gracilis tendon graft is passed through two converging patellar tunnels: the first tunnel directed from medial to lateral and the second from anterior to posterior. The graft ends are looped around adductor tendon and sutured together. The graft is then sutured to the adductor tendon and proximal to MCL insertion. ${ }^{25}$

\section{Pedicled Graft Techniques}

With goal of minimizing tunnel drilling on the patella and femur and reducing the risk of growth plate injury, different techniques were described with pedicled grafts. Preserving one graft tendon insertion minimizes the fixation on that specific site (patella or femur).

\section{Pedicled Quadriceps Tendon Graft}

Fink et $\mathrm{al}^{26}$ (- Fig. 3A) described a minimally invasive MPFL reconstruction using a quadriceps tendon (QT) strip. With a $3-\mathrm{cm}$ incision on the superomedial pole of the patella, an $8-10 \mathrm{~cm}$ central strip of the QT is harvested and left attached distally. Elevating the prepatellar tissue, a soft tissue tunnel from the superomedial border of the patella to the medial edge of the QT is created. The graft is then passed into the tunnel and fixed to the medial retinaculum with resorbable 2-0 sutures. A second incision is made over the femoral attachment of the MPFL and a $2.4-\mathrm{mm}$ guide pin is drilled under fluoroscopic guidance. The guide pin is overdrilled with a 6-mm cannulated reamer to a depth of $30 \mathrm{~mm}$. Fixation is achieved with a resorbable interference screw at 20 degrees of flexion. In children with open physes, the graft is fixed with one suture anchor or sutures alone. ${ }^{26}$
Abouelsoud et $\mathrm{al}^{27}$ ( - Fig. 3B) described a similar technique with the medial third of the QT. The medial retinaculum is opened $5 \mathrm{~mm}$ from the medial border of the patella starting at the proximal pole down to the distal pole. The retinaculum is separated from the capsule down to the medial epicondyle. The tendon is then turned under the medial retinaculum. With the patella centered in the groove, plication of the medial retinaculum is then performed with no. 2 resorbable sutures at three specific points: upper medial (vastus medialis obliquus), mid-medial (MPFL), and distal medial (medial patellotibial ligament [MPTL]). The QT is then fixed to two points: (1) to the thick periosteum of the MPFL anatomic attachment (found with fluoroscopy, with the Schottle's method $^{9}$ ) with no. 2 nonabsorbable, braided sutures; and (2) to the adductor magnus tendon. ${ }^{27}$

Nelitz et $\mathrm{al}^{28,29}$ (- Fig. 3C) described a similar pedicled quadriceps technique. A medial third of the QT is harvested (preserving a thin layer of the medial tendon for closure) and left attached to the patella. The graft is then passed between the capsule and the medial retinaculum. The graft is fixed at anatomic femoral insertion of the MPFL in a bone socket with an interference screw. Angulation of the femoral tunnel 15 to 20 degrees both distally and anteriorly minimizes damage to the physis, notch, and distal femoral cartilage. The graft was then secured to the patella with subperiosteal braided resorbable suture. ${ }^{28,29}$

\section{Pedicled Hamstring Tendons Graft}

Historically, Galeazzi ${ }^{30}$ ( - Fig. 4A) in 1922 described one of the first pedicled hamstring reconstruction. The semitendinosus tendon is harvested and an oblique patellar tunnel is created (from the inferomedial to the superolateral aspect of the patella). A complete lateral release is performed. The graft is inserted in the tunnel and then sutured to the periosteum of the patella and, if sufficient length is available, to itself. ${ }^{30}$

Currently, when pedicled hamstrings are used, usually both MPFL and MPTL reconstruction is performed.

In 2009, Nietosvaara et $\mathrm{al}^{31}$ described a MPFL and MPTL reconstruction technique using the semitendinosus tendon, harvested with a tendon stripper with 3-cm approach to the pes anserinus. The semitendinosus distal insertion is preserved. Two additional 2-cm incisions are made at the inferomedial and
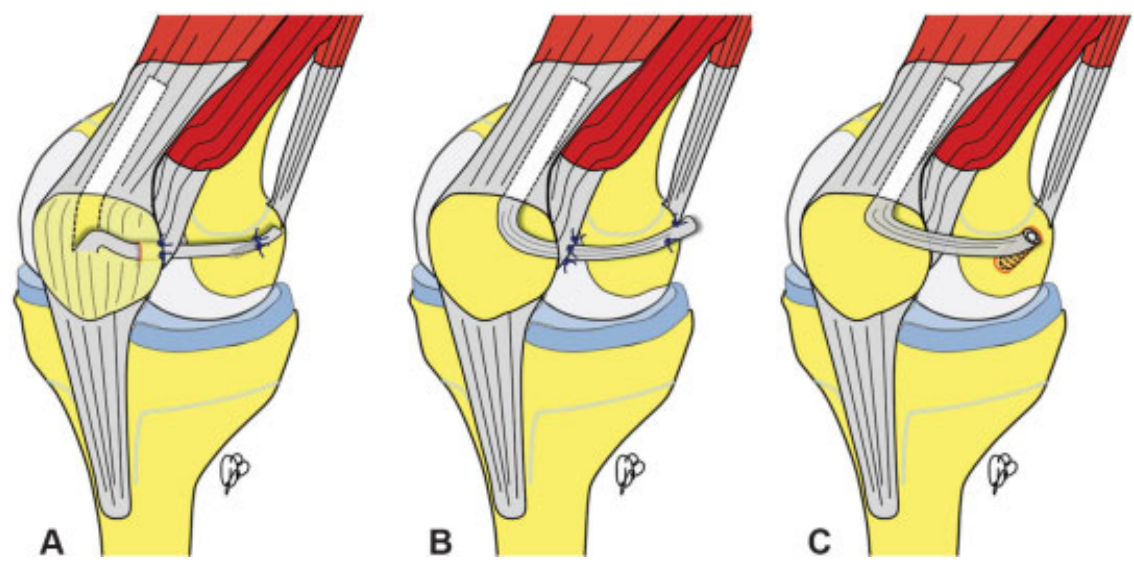

Fig. 3 Pedicled quadriceps graft techniques: (A) Fink's technique; (B) Abouelsoud's technique; and (C) Nelitz's technique. 

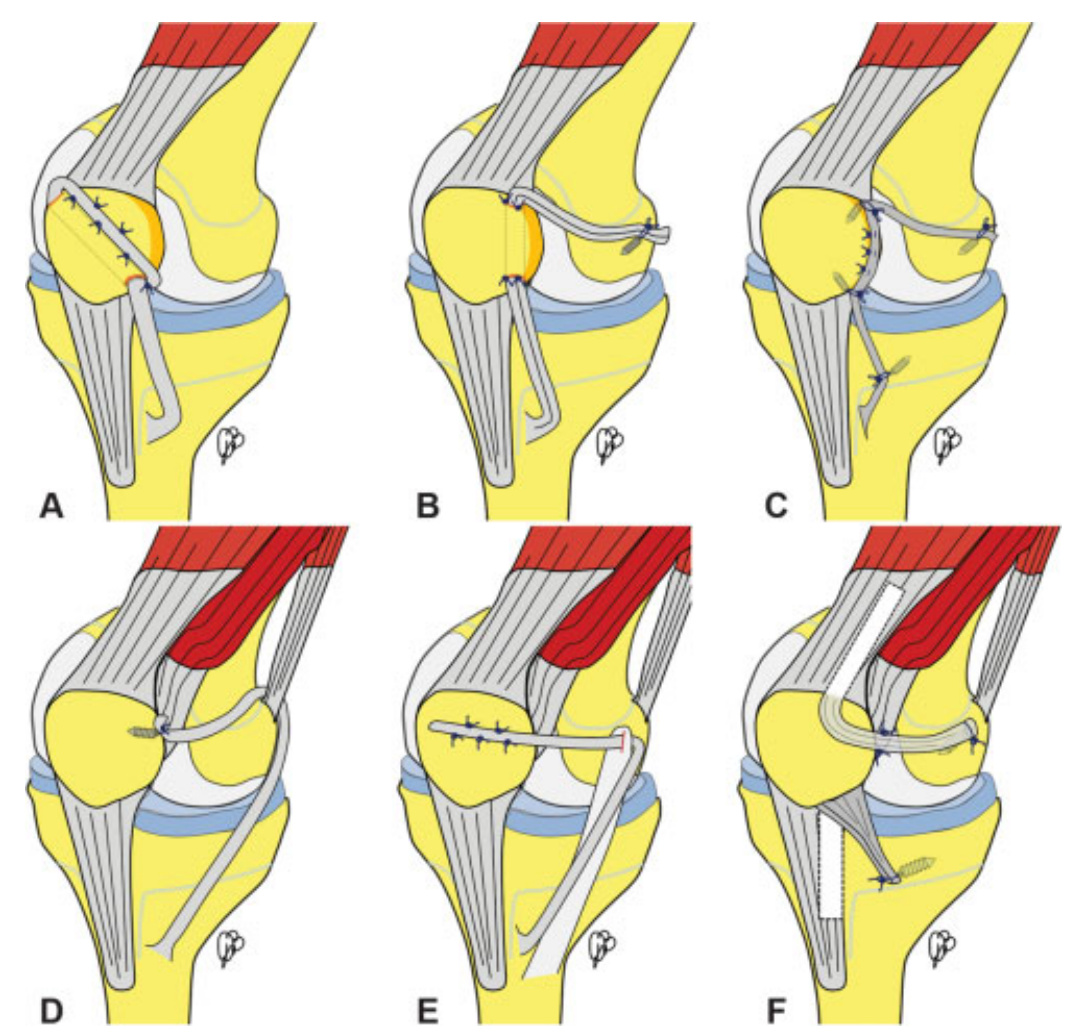

Fig. 4 Pedicled hamstring graft techniques: (A) Galeazzi's technique; (B) Giordano's technique; (C) Sadigursky's technique; (D) Alm's technique; and (E) Deie's technique. Pedicled quadriceps plus pedicled patellar tendon technique: (F) Hinckel's technique.

superomedial borders of the patella. A 4-mm longitudinal tunnel is created in the medial quadrant of the patella. The graft is passed below the fascia and retrieved from the inferomedial incision. The graft is then passed through the tunnel from distal to proximal, exiting from the superomedial pole. The tendon graft is tunneled in a subfascial plane to the adductor tubercle, where a $3-\mathrm{cm}$ incision is made. The graft is tensioned with the knee in 30 to 45 degrees of flexion. Then, the graft is fixed at the adductor tubercle with an interference screw. ${ }^{31}$

Giordano et $\mathrm{al}^{32}$ described a variation of this technique ( - Fig. 4B). The authors used both semitendinosus and gracilis tendons, preserving the distal insertion. A patellar tunnel is drilled as described by Nietosvaara et al. ${ }^{31}$ The graft is then passed through the patellar tunnel, tensioned with the patella correctly seated in the trochlea, and sutured to distal patellar periosteum. The graft is then tensioned and fixed at the adductor tubercle with a suture anchor. At the end, the graft is secured at the proximal pole of the patella with bioabsorbable sutures. ${ }^{32}$

In 2017, Sadigursky et $\mathrm{al}^{33}$ (- Fig. 4C) described a variation of Giordano's ${ }^{32}$ and Nietosvaara's ${ }^{31}$ techniques. The semitendinosus tendon only is harvested and left attached distally. The graft is fixed with a suture anchor to the anteromedial tibia, proximal to the physis, and forming a 20-degree angle with patellar tendon. Two suture anchors are then placed at the distal and proximal thirds of the medial patellar aspect. A fourth anchor is then positioned with the Schottle's method ${ }^{9}$ on the anatomical MPFL femoral insertion, distal to the femoral physis. The graft is fixed at the inferomedial patella at
90 degrees of the flexion, secured with sutures to the medial patellar border, fixed again at the third suture anchor, and finally fixed at the femur at 45 to 60 degrees of flexion. ${ }^{33}$

Alm et $\mathrm{al}^{34}$ ( - Fig. 4D) described a modified adductor sling technique with a pedicled semitendinosus or gracilis tendon graft, with preserved distal insertion. The free end of the graft, passed around the adductor tendon from posterior to anterior, is fixed with a suture anchor at the medial facet of the patella.

In Deie et al's technique ${ }^{35}$ (- Fig. 4E), a pedicled semitendinosus autograft is used, preserving the distal insertion. Through an incision 2-cm long over the femoral attachment of the MCL, a $1-\mathrm{cm}$ slit is made in its posterior one-third to act as a pulley, and through a curved incision over the patella the semitendinosus tendon is transferred through the pulley to the patella. The site of the pulley is adjacent to the anatomical attachment of the MPFL on the femur. The graft is sutured to the anterior surface of the patella with the knee flexed at 30 degrees. ${ }^{35}$

\section{Combined Pedicled Quadriceps and Patellar Tendon Grafts}

Hinckel et $\mathrm{al}^{36}$ (- $\mathbf{\text { Fig. }}$ 4F) described a MPFL and MPTL reconstruction using both pedicled QT and patellar tendon grafts, respectively. Two small anterior incisions $(2-3 \mathrm{~cm}$ each) are made to harvest the quadricipital and patellar grafts (alternatively an anterior $8-\mathrm{cm}$ incision can be performed), together with an incision $(2 \mathrm{~cm})$ on the medial epicondyle. An $8-\mathrm{cm}$ wide medial QT strip and a $6-\mathrm{mm}$ wide medial patellar tendon strip are harvested and left 
attached to the patella. The patellar graft is fixed with a 3.5 - or 5-mm suture anchor, placed proximal to the growth plate $(2 \mathrm{~cm}$ distal to the joint line and $2 \mathrm{~cm}$ medial to the tibial tubercle), with the knee flexed at 90 degrees. Then, the quadriceps graft is passed between layers 2 and 3 and fixed anatomically (Schottle's method $^{9}$ ) on the femur with a 3.5- or 5-mm suture anchor, with the knee at 30 to 45 degrees of flexion. Then, two or three sutures are placed between the quadricipital graft and the vastus medialis for a dynamic active component. ${ }^{36}$

\section{Pedicled Adductor Magnus}

To avoid hardware placement or tunnel drilling close to the distal femoral growth plate, some authors described different MPFL reconstruction techniques with a pedicled adductor magnus graft.

In the papers by Avikainen et $\mathrm{al}^{37}$ and Malecki et $\mathrm{al}^{38}$ (-Fig. 5A), a $10-\mathrm{cm}$ medial incision is made and the vastus medialis muscle is elevated anteriorly. The adductor magnus tendon is identified and harvested, preserving the distal insertion. After creating a tunnel in the patella, the tendon is inserted in the tunnel and sutured on the medial and lateral side of the patella, with no. 1 braided absorbable sutures.

Steiner et $\mathrm{al}^{39}$ ( - Fig. 5B) described a modification of this technique. With the goal of achieving a more anatomical femoral position, the adductor tendon graft is passed through a short femoral tunnel, exiting on the anatomical MPFL insertion. The graft is then passed through the patella, as previously described, and sutured to the anterior aspect of the patella or to itself, if long enough.

\section{Pedicled Patellar Tendon}

With an increased $Q$ angle ( $>15$ degrees) a distal realignment should be considered. However, in the pediatric population, tibial tubercle transfer places the tibial tubercle growth plate at risk. $^{3}$ For this reason, different soft tissue distal realignment techniques (mostly with a pedicled patellar tendon) have been described.

The Roux-Goldthwait procedure (-Fig. 6A) was originally described by Roux ${ }^{40}$ in 1888 and subsequently modified by
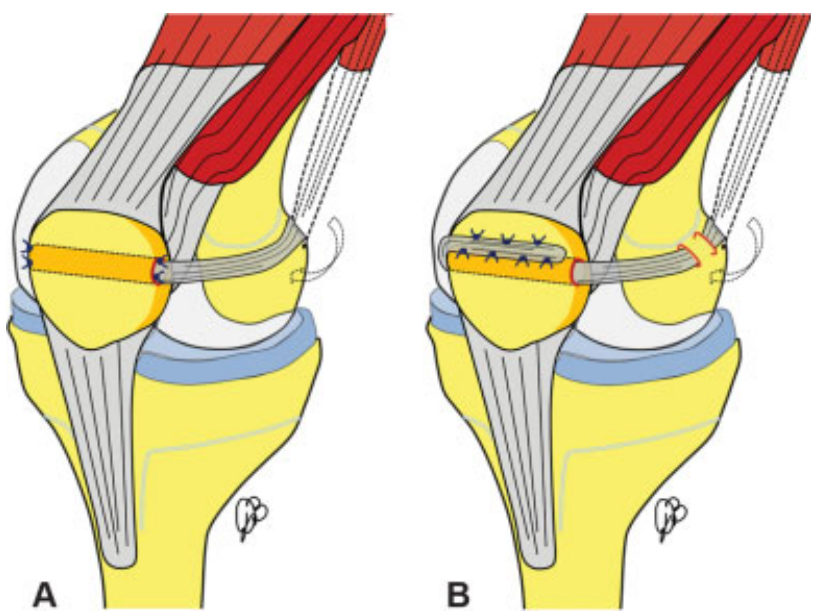

Fig. 5 Pedicled adductor magnus graft techniques: (A) Avikainen's and Malecki's techniques; and (B) Steiner's technique.
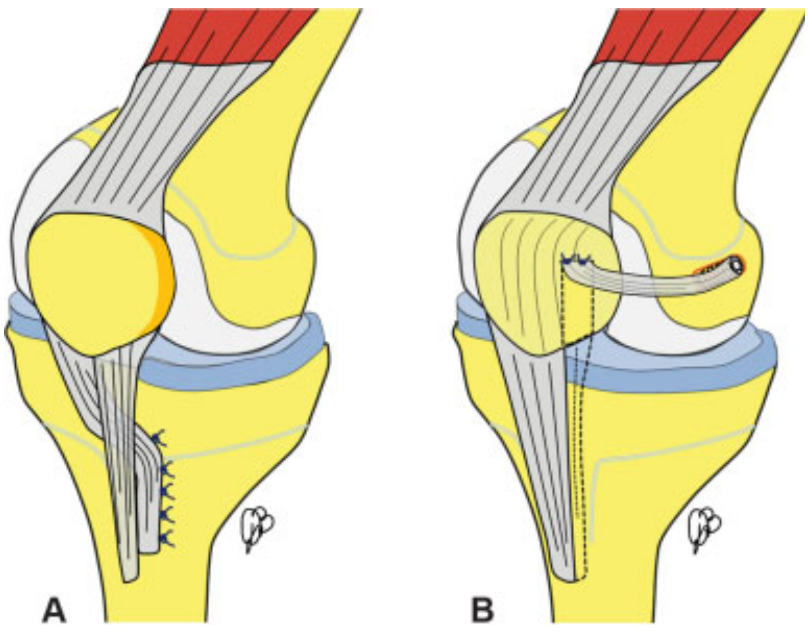

Fig. 6 Pedicled patellar tendon graft techniques: (A) Roux-Goldthwait technique; and (B) Bitar's technique.

Goldthwait ${ }^{41}$ in 1899 . The procedure involves splitting the patellar tendon longitudinally and detaching the lateral half from the tibial tuberosity distally. The lateral half is then transferred beneath the intact medial half and sutured to the soft tissues and periosteum on the medial side of the tibial epiphysis. $^{3}$

Luhmann et $\mathrm{al}^{42}$ described a technique for medialization of the patellar tendon in skeletally immature patients. The tendon is completely detached from the tibial tubercle, medialized approximately $50 \%$ of its width, and then sutured to the periosteum.

Bitar et $\mathrm{al}^{43}$ (- Fig. 6B) described a technique using a pedicled medial patellar tendon. An anteromedial longitudinal incision is made starting from the midpoint between superomedial patella border and medial epicondyle and ending on the medial aspect of the tibial tubercle. The medial third of the patellar tendon is detached distally. Proximally the tendon is carefully separated from the patella up to the transition between the proximal third and medial third of the patella. At this point, the graft is still attached to the patella. The attachment is reinforced with nonabsorbable sutures, before rotating the graft. The femoral insertion of the MPFL is identified, a tunnel is created, and the graft fixed to the tunnel with an interference screw. ${ }^{43}$

\section{Complications}

Complications of stabilization procedures for patellofemoral instability in skeletally immature patients include: redislocation, stiffness, patellofemoral pain, patellofemoral arthritis, and growth disturbance. Parikh et $\mathrm{al}^{44}$ retrospectively reviewed 179 knees after MPFL reconstruction. The authors described 38 complications in 29 knees (16.2\%), with 34 major and 4 minor. Major complications included recurrent lateral patellar instability (8 patients), knee motion stiffness with flexion deficits (8 patients), patellar fractures (6 patients), and patellofemoral arthrosis/pain (5 patients). Eighteen of 38 (47\%) complications were secondary to 
technical factors and were considered preventable. Female sex and bilateral MPFL reconstructions were risk factors associated with postoperative complications. ${ }^{44}$

Complications could be related to the femoral insertion of the neo-ligament. A femoral tunnel too proximal and anterior may cause medial patellofemoral articular overload, iatrogenic medial subluxation, or recurrent lateral instability due to stretching of the graft. ${ }^{45}$ If the femoral tunnel is too distal or posterior, excessive graft tensioning in extension, extensor lag, or graft stretching are possible consequences. ${ }^{44}$

A $1.2 \%$ pooled total risk of recurrent instability after isolated MPFL reconstruction with a reoperation risk of $3.1 \%$ was reported by Schneider et al. ${ }^{46}$

Case reports of growth disturbances after MPFL reconstruction are available in the literature. Seitlinger et $\mathrm{al}^{47}$ reported a case of acquired femoral flexion deformity due to physeal injury after MPFL reconstruction. A 14-year-old female presented an extension deficit ( $\sim 20$ degrees) and recurrent patellar dislocation after MPFL reconstruction. At the magnetic resonance imaging, it was evident that a femoral screw breached the dorsomedial femoral physis. An open-wedge extension osteotomy of the distal femur, medialization of the tibial tubercle, and deepening trochleoplasty were necessary to correct this deformity. After 12 months, the patient had no recurrent dislocations or instability signs. ${ }^{47}$

To reduce complications, the femoral insertion of the graft has to be found under fluoroscopic guidance, according to the Schottle's $\operatorname{method}^{9}$ on the perfect lateral view of the knee. In skeletally immature patients, an AP view is also mandatory to prevent physeal injuries.

Patellar fractures are also potential complications. To reduce this risk, a single patellar tunnel (or no tunnel) fixation should be used. ${ }^{44}$

\section{Conclusion}

Since this article was intended to be a reference guide of the available surgical techniques for MPFL reconstruction and nonanatomic patellofemoral stabilization through a narrative review, no significant conclusions could be drawn regarding the superiority of one technique over the others. However, we believe that anatomical MPFL reconstruction should be preferred to nonanatomic techniques. In patients with open physes, the distal femoral growth plate should always be preserved according to the tips described by different authors and reported in this review. ${ }^{10,11,13}$ In case of significant factors predisposing to instability (i.e., rotational deformities, patella alta, marked valgus deformity), these should be corrected whenever possible (i.e., temporary hemiepiphysiodesis for severe valgus deformity, shortening of the patellar tendon for patella alta); however, since medialization (and/or distalization) tibial tubercle osteotomies and trochleoplasty are contraindicated in skeletally immature patients, these are the most challenging cases. Some authors suggest that, in these cases, a soft tissue distal realignment (according to one of the techniques described) should be performed together with anatomic MPFL reconstruction. However, there is not enough evidence to support this concept. Some authors advocate the use of isolated soft tissue buy-time procedures. However, counseling regarding second-stage definitive surgery after the end of growth is mandatory. ${ }^{48}$

Further studies are required to identify the most reliable surgical techniques in these challenging cases.

\section{Conflict of Interest}

D.B. and R.R. report other from Zimmer Biomet, outside the submitted work. All the other authors report no conflict of interest.

\section{References}

1 Vavken P, Wimmer MD, Camathias C, Quidde J, Valderrabano V, Pagenstert G. Treating patella instability in skeletally immature patients. Arthroscopy 2013;29(08):1410-1422

2 Khormaee S, Kramer DE, Yen YM, Heyworth BE. Evaluation and management of patellar instability in pediatric and adolescent athletes. Sports Health 2015;7(02):115-123

3 Weeks KD III, Fabricant PD, Ladenhauf HN, Green DW. Surgical options for patellar stabilization in the skeletally immature patient. Sports Med Arthrosc Rev 2012;20(03):194-202

4 Beaufils P, Thaunat M, Pujol N, Scheffler S, Rossi R, Carmont M. Trochleoplasty in major trochlear dysplasia: current concepts. Sports Med Arthrosc Rehabil Ther Technol 2012;4:7

5 Hensler D, Sillanpaa PJ, Schoettle PB. Medial patellofemoral ligament: anatomy, injury and treatment in the adolescent knee. Curr Opin Pediatr 2014;26(01):70-78

6 Warren LF, Marshall JL. The supporting structures and layers on the medial side of the knee: an anatomical analysis. J Bone Joint Surg Am 1979;61(01):56-62

7 Alaia MJ, Cohn RM, Strauss EJ. Patellar instability. Bull Hosp Jt Dis (2013) 2014;72(01):6-17

8 LaPrade RF, Engebretsen AH, Ly TV, Johansen S, Wentorf FA, Engebretsen L. The anatomy of the medial part of the knee. J Bone Joint Surg Am 2007;89(09):2000-2010

9 Schottle PB, Romero J, Schmeling A, Weiler A. Technical note: anatomical reconstruction of the medial patellofemoral ligament using a free gracilis autograft. Arch Orthop Trauma Surg 2008;128 (05):479-484

10 Nelitz M, Dornacher D, Dreyhaupt J, Reichel H, Lippacher S. The relation of the distal femoral physis and the medial patellofemoral ligament. Knee Surg Sports Traumatol Arthrosc 2011;19(12): 2067-2071

11 Shea KG, Grimm NL, Belzer J, Burks RT, Pfeiffer R. The relation of the femoral physis and the medial patellofemoral ligament. Arthroscopy 2010;26(08):1083-1087

12 Farrow LD, Alentado VJ, Abdulnabi Z, Gilmore A, Liu RW. The relationship of the medial patellofemoral ligament attachment to the distal femoral physis. Am J Sports Med 2014;42(09):2214-2218

13 Nguyen CV, Farrow LD, Liu RW, Gilmore A. Safe drilling paths in the distal femoral epiphysis for pediatric medial patellofemoral ligament reconstruction. Am J Sports Med 2017;45(05):1085-1089

14 Kepler CK, Bogner EA, Hammoud S, Malcolmson G, Potter HG, Green DW. Zone of injury of the medial patellofemoral ligament after acute patellar dislocation in children and adolescents. Am J Sports Med 2011;39(07):1444-1449

15 Lewallen LW, McIntosh AL, Dahm DL. Predictors of recurrent instability after acute patellofemoral dislocation in pediatric and adolescent patients. Am J Sports Med 2013;41(03):575-581

16 Nwachukwu BU, So C, Schairer WW, Green DW, Dodwell ER. Surgical versus conservative management of acute patellar dislocation in children and adolescents: a systematic review. Knee Surg Sports Traumatol Arthrosc 2016;24(03):760-767

17 Hohn E, Pandya NK. Does the utilization of allograft tissue in medial patellofemoral ligament reconstruction in pediatric and 
adolescent patients restore patellar stability? Clin Orthop Relat Res 2017;475(06):1563-1569

18 Nelitz M, Reichel H, Dornacher D, Lippacher S. Anatomical reconstruction of the medial patellofemoral ligament in children with open growth-plates. Arch Orthop Trauma Surg 2012;132(11): $1647-1651$

19 Ladenhauf HN, Berkes MB, Green DW. Medial patellofemoral ligament reconstruction using hamstring autograft in children and adolescents. Arthrosc Tech 2013;2(02):e151-e154

20 Shafizadeh S, Balke M. Medial patellofemoral ligament reconstruction: a new technique for graft fixation at the patella without implants. Arthrosc Tech 2014;3(01):e115-e117

21 Sadigursky D, de Melo Laranjeira MS, Nunes M, Caneiro RJ, Colavolpe PO. Reconstruction of the medial patellofemoral ligament by means of the anatomical double-bundle technique using metal anchors. Rev Bras Ortop 2016;51(03):290-297

22 Kumahashi N, Kuwata S, Tadenuma T, Kadowaki M, Uchio Y. A "sandwich" method of reconstruction of the medial patellofemoral ligament using a titanium interference screw for patellar instability in skeletally immature patients. Arch Orthop Trauma Surg 2012;132(08):1077-1083

23 Yercan HS, Erkan S, Okcu G, Ozalp RT. A novel technique for reconstruction of the medial patellofemoral ligament in skeletally immature patients. Arch Orthop Trauma Surg 2011;131(08):1059-1065

24 Gomes JE. Comparison between a static and a dynamic technique for medial patellofemoral ligament reconstruction. Arthroscopy 2008;24(04):430-435

25 Lind M, Enderlein D, Nielsen T, Christiansen SE, Faunø P. Clinical outcome after reconstruction of the medial patellofemoral ligament in paediatric patients with recurrent patella instability. Knee Surg Sports Traumatol Arthrosc 2016;24(03):666-671

26 Fink C, Veselko M, Herbort M, Hoser C. Minimally invasive reconstruction of the medial patellofemoral ligament using quadriceps tendon. Arthrosc Tech 2014;3(03):e325-e329

27 Abouelsoud MM, Abdelhady A, Elshazly O. Anatomic physealsparing technique for medial patellofemoral ligament reconstruction in skeletally immature patients with ligamentous laxity. Eur J Orthop Surg Traumatol 2015;25(05):921-926

28 Nelitz M, Dreyhaupt J, Williams SRM. Anatomic reconstruction of the medial patellofemoral ligament in children and adolescents using a pedicled quadriceps tendon graft shows favourable results at a minimum of 2-year follow-up. Knee Surg Sports Traumatol Arthrosc 2018;26(04):1210-1215

29 Nelitz M, Williams SRM. Anatomic reconstruction of the medial patellofemoral ligament in children and adolescents using a pedicled quadriceps tendon graft. Arthrosc Tech 2014;3(02):e303-e308

30 Galeazzi R. Nuove applicazioni del trapianto muscolare e tendineo. Arch Di Ortop Milano 1922;38:315-323

31 Nietosvaara Y, Paukku R, Palmu S, Donell ST. Acute patellar dislocation in children and adolescents. Surgical technique. J Bone Joint Surg Am 2009;91(Suppl 2 Pt 1):139-145

32 Giordano M, Falciglia F, Aulisa AG, Guzzanti V. Patellar dislocation in skeletally immature patients: semitendinosous and gracilis augmentation for combined medial patellofemoral and medial patellotibial ligament reconstruction. Knee Surg Sports Traumatol Arthrosc 2012;20(08):1594-1598
33 Sadigursky D, Garcia LC, Armede M, Oliveira LR, Carneiro RJF, Colavolpe PO. Medial patellofemoral ligament and medial patellotibial ligament reconstruction in children: preliminary results. Rev Bras Ortop 2017;52(04):417-422

34 Alm L, Krause M, Mull C, Frosch KH, Akoto R. Modified adductor sling technique: a surgical therapy for patellar instability in skeletally immature patients. Knee 2017;24(06):1282-1288

35 Deie M, Ochi M, Sumen Y, Yasumoto M, Kobayashi K, Kimura H. Reconstruction of the medial patellofemoral ligament for the treatment of habitual or recurrent dislocation of the patella in children. J Bone Joint Surg Br 2003;85(06):887-890

36 Hinckel BB, Gobbi RG, Demange MK, Bonadio MB, Pécora JR, Camanho GL. Combined reconstruction of the medial patellofemoral ligament with quadricipital tendon and medial patellotibial ligament with patellar tendon. Arthrosc Tech 2016;5(01): e79-e84

37 Avikainen VJ, Nikku RK, Seppänen-Lehmonen TK. Adductor magnus tenodesis for patellar dislocation. Technique and preliminary results. Clin Orthop Relat Res 1993;(297):12-16

38 Malecki K, Fabis J, Flont P, Lipczyk Z, Niedzielski K. Preliminary results of two surgical techniques in the treatment of recurrent patellar dislocation: medial patellofemoral ligament reconstruction versus combined technique of vastus medialis advancement, capsular plasty and Roux-Goldthwait procedure in treatment of recurrent patellar dislocation. Int Orthop 2016;40(09):1869-1874

39 Steiner TM, Torga-Spak R, Teitge RA. Medial patellofemoral ligament reconstruction in patients with lateral patellar instability and trochlear dysplasia. Am J Sports Med 2006;34(08):1254-1261

40 Roux C. Recurrent dislocation of the patella: operative treatment. 1888. Clin Orthop Relat Res 2006;452(452):17-20

41 Goldthwait JE. Dislocation of the patella. Trans Am Orthop Assoc 1895;8:237-238

42 Luhmann SJ, O'Donnell JC, Fuhrhop S. Outcomes after patellar realignment surgery for recurrent patellar instability dislocations: a minimum 3-year follow-up study of children and adolescents. J Pediatr Orthop 2011;31(01):65-71

43 Bitar AC, Demange MK, D'Elia CO, Camanho GL. Traumatic patellar dislocation: nonoperative treatment compared with MPFL reconstruction using patellar tendon. Am J Sports Med 2012;40(01): 114-122

44 Parikh SN, Nathan ST, Wall EJ, Eismann EA. Complications of medial patellofemoral ligament reconstruction in young patients. Am J Sports Med 2013;41(05):1030-1038

45 Bollier M, Fulkerson J, Cosgarea A, Tanaka M. Technical failure of medial patellofemoral ligament reconstruction. Arthroscopy 2011;27(08):1153-1159

46 Schneider DK, Grawe B, Magnussen RA, et al. Outcomes after isolated medial patellofemoral ligament reconstruction for the treatment of recurrent lateral patellar dislocations: a systematic review and meta-analysis. Am J Sports Med 2016;44(11): 2993-3005

47 Seitlinger G, Moroder P, Fink C, Wierer G. Acquired femoral flexion deformity due to physeal injury during medial patellofemoral ligament reconstruction. Knee 2017;24(03):680-685

48 Hasler CC, Studer D. Patella instability in children and adolescents. EFORT Open Rev 2017;1(05):160-166 